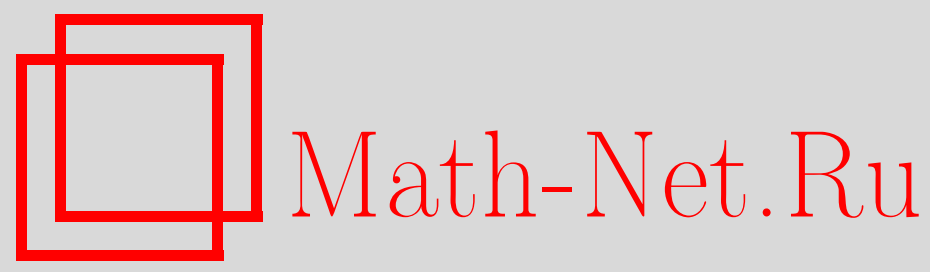

В. С. Климов, Типовые числа критических точек негладких функционалов, Матем. заметки, 2002, том 72, выпуск 5, 693-705

DOI: https://doi.org/10.4213/mzm458

Использование Общероссийского математического портала Math-Net.Ru подразумевает, что вы прочитали и согласны с пользовательским соглашением http://www.mathnet.ru/rus/agreement

Параметры загрузки:

IP: 18.234 .156 .22

26 апреля 2023 г., $13: 14: 39$

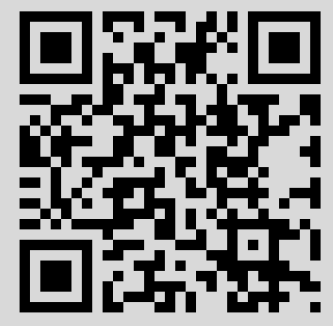




\section{ТИПОВЫЕ ЧИСЛА КРИТИЧЕСКИХ ТОЧЕК НЕГЛАДКИХ ФУНКЦИОНАЛОВ}

\section{В. С. Климов}

Изучаются типовые числа критических точек липшицевых функционалов. Устанавливаются варианты неравенств Морса; показывается, что топологический индекс изолированной критической точки равен альтернированной сумме ее типовых чисел. Приводятся формулы для вычисления типовых чисел нулевой критической точки одного функционала.

Библиография: 18 названий.

Введение. Статья посвящена обобщению теории Морса на негладкие функционалы. Первоначально бесконечномерные версии результатов Морса доказьвались для класса $C^{2}$ дважды непрерывно дифференцируемых функционалов (см., например, [1]-[5] и приведенную там библиографию). Развитая теория оказалась неприменимой к интегральным функционалам многомерного вариационного исчисления, принадлежащим $C^{2}$ лишь при существенных дополнительньх ограничениях. В связи с этим в [3] предложен вариант теории Морса для функционалов класса $C^{1,1}$. Ниже порядок гладкости снижается (по сравнению с [3]) на единицу; основные результаты новы и для функций конечного числа переменных. В бесконечномерном случае существенно то, что рассматриваемые банаховы пространства не предполагаются гильбертовыми. Наиболее значительные из возникающих здесь трудностей связаны с построением деформаций, уменьшаюших значения функционала. Для преодоления этих трудностей используются идеи теории монотонных операторов [3], [5]-[7], результаты работ [8], [9], методы нелинейного анализа [10]-[15].

В п. 1 статьи изучаются типовые числа критических значений липшищевых функционалов, определенных на выпуклом замкнутом подмножестве сепарабельного рефлексивного пространства. Устанавливаются варианты неравенств Морса (теорема 2). В п. 2 вводятся типовые числа критических точек. Показывается, что топологический индекс изолированной критической точки равен альтернированной сумме ее типовых чисел (теорема 4). Пункт 3 содержит формулу для вычисления типовых чисел нулевой критической точки однородного функционала (теорема 5 ).

Используются обозначения: $\mathfrak{\mathfrak { M }}(\mathfrak{M}, \partial \mathfrak{M})$ - замькание (внутренность, граница) подмножества $\mathfrak{M}$ метрического пространства $(\mathfrak{R}, \rho), \theta_{\mathfrak{R}}(\mathfrak{N}, \mathfrak{M})=\sup _{x \in \mathfrak{N}} \inf _{y \in \mathfrak{M}} \rho(x, y)-$ уклонение множества $\mathfrak{N} \subset \mathfrak{R}$ от множества $\mathfrak{M} \subset \mathfrak{R}$ в метрике $\rho ; X$ и $X^{*}$ - действительное банахово пространство и сопряженное к нему соответственно, $\langle\cdot, \cdot\rangle-$ каноническая билинейная форма на $X \times X^{*}, s\left(x^{*}, \mathscr{M}\right)=\sup \left\{\left\langle x, x^{*}\right\rangle, x \in \mathscr{M}\right\}\left(x^{*} \in X^{*}\right)$ - опорная 
функция множества $\mathscr{M} \subset X, G(X)$ - совокупность всех непустых выпуклых замкнутых подмножеств пространства $X$. Если $x \in Q, Q \in G(X)$, то множества, определяемые соотношениями

$$
T_{Q}(x)=\overline{\bigcup_{\lambda>0} \lambda(Q-x)}, \quad N_{Q}(x)=\left\{x^{*} \in X^{*},\left\langle v-x, x^{*}\right\rangle \leqslant 0 \forall v \in Q\right\},
$$

назьвают касательными и нормальньми конусами к множеству $Q$ в точке $x$. Далее, $\Lambda(\mathscr{M}, Y)$ - совокупность отображений множества $\mathscr{M} \subset X$ в банахово пространство $Y$, удовлетворяющих локальному условию Липшица; $\Lambda(\mathscr{M})=\Lambda(\mathscr{M} ; \mathbb{R})$.

Многозначное отображение $\mathscr{F}$ множества $\mathscr{M}_{1}$ во множество $\mathscr{M}_{2}$ - это оператор, ставящий в соответствие элементу $x$ из $\mathscr{M}_{1}$ непустое множество $\mathscr{F}(x) \subset \mathscr{M}_{2} ;$ если $\mathscr{M} \subset \mathscr{M}_{1}$, то $\mathscr{F}(\mathscr{M})=\cup \mathscr{F}(x)(x \in \mathscr{M})$ - область значений отображения $\mathscr{F}$ на множестве $\mathscr{M}$. Отображение $\mathscr{F}: \mathscr{M}_{1} \rightarrow \mathscr{M}_{2}\left(\mathscr{M}_{1}, \mathscr{M}_{2}\right.$ - подмножества метрических пространств $\mathfrak{R}_{1}, \mathfrak{R}_{2}$ соответственно) назьвают ограниченным, если для каждого ограниченного множества $\mathscr{M} \subset \mathscr{M}_{1}$ область значений $\mathscr{F}(\mathscr{M})$ есть ограниченное подмножество $\mathfrak{R}_{2}$.

1. Типовые числа критических значений. Всюду далее $X$ - сепарабельное рефлексивное банахово пространство, $\|\cdot\|$ - норма в $X$, символы $\rightarrow$ и $\rightarrow$ означают слабую и сильную сходимости соответственно; $Q \in G(X), X_{0}=\operatorname{Lin}(Q-Q)$ - линейная оболочка множества $Q-Q$; ниже предполагается, что $\bar{X}_{0}=X ; \Gamma(Q)-$ совокупность таких конечномерньх подпространств $E$ пространства $X$, что внутренность $\operatorname{ri}_{E}(Q \cap E)$ множества $Q \cap E$ относительно пространства $E$ непуста.

Пусть $\mathscr{M}=\overline{\mathscr{M}} \subset Q$. Обозначим через $S(\mathscr{M})$ класс ограниченных многозначных отображений $\mathscr{F}: \mathscr{M} \rightarrow G\left(X^{*}\right)$, удовлетворяющих условию: для произвольных последовательностей $x_{n} \in \mathscr{M}, x_{n}^{*} \in \mathscr{F}\left(x_{n}\right)$, обладаюших свойствами

$$
x_{n} \rightarrow x, \quad x_{n}^{*} \rightarrow x^{*}, \quad \varlimsup_{n \rightarrow \infty}\left\langle x_{n}, x_{n}^{*}\right\rangle \leqslant\left\langle x, x^{*}\right\rangle,
$$

имеют место соотношения

$$
x_{n} \rightarrow x, \quad x^{*} \in \mathscr{F}(x) .
$$

Близкиек $S(\mathscr{M})$ классы однозначных операторов рассматривались в [2], [3], [5]-[7]. Примеры многозначных операторов класса $S(\mathscr{M})$ можно найти в [8], [9].

Элемент $x \in \mathscr{M}$ назовем особой точкой оператора $\mathscr{F}$ класса $S(\mathscr{M})$, если

$$
\left\langle v-x, x^{*}\right\rangle \geqslant 0 \quad \forall v \in Q
$$

для некоторого $x^{*}$ из $\mathscr{F}(x)$. Соотношение $(3)$ означает, что особые точки оператора $\mathscr{F}$ являются решениями соответствующего вариационного неравенства [10]. Оператор $\mathscr{F}$ класса $S(\mathscr{M})$ назовем невырожденным на множестве $\mathfrak{M} \subset \mathscr{M}$, если $\mathfrak{M}$ не содержит особых точек $\mathscr{F}$.

ПРЕДЛОЖЕНИЕ 1 [8]. Пусть оператор $\mathscr{F}$ класса $S(Q)$ невырожден на ограниченном замкнутом множестве $\mathfrak{M} \subset Q$. Тогда существуют $E$ из $\Gamma(Q)$, ограниченное отображсение $w$ класса $\Lambda(Q, X)$ и постоянная $\delta>0$, обладающие свойствами

$$
w(x) \in \operatorname{ri}_{E}(Q \cap E) \quad \forall x \in Q, \quad s(w(x)-x, \mathfrak{F}(x))<-\delta \quad \forall x \in \mathfrak{M} .
$$


Пусть $\Omega$ - ограниченное открытое (в относительной топологии) подмножество множества $Q$, рассматриваемого как метрическое пространство с метрикой $\rho(x, y)=\|x-y\|$, $\partial_{Q} \Omega$ - относительная граница множества $\Omega$. Если оператор $\mathscr{F}$ класса $S(Q)$ невырожден на $\partial_{Q} \Omega$, то согласно предложению 1 существуют пространство $E$ класса $\Gamma(Q)$ и векторное поле $w$ класса $\Lambda(Q, E)$ такие, что $w(x) \in \operatorname{ri}_{E}(Q \cap E) \forall x \in Q,\left\langle x-w(x), x^{*}\right\rangle>0$ $\left(x \in \partial_{Q} \Omega, x^{*} \in \mathscr{F}(x)\right)$. В частности, поле $(I-w)(x)=x-w(x)$ невырождено на $\partial_{Q} \Omega$ и определено относительное вращение $\gamma\left(I-w, \partial_{Q} \Omega\right)$ поля $I-w$ на $\partial_{Q} \Omega[11]$, [12]. Число $\gamma\left(I-w, \partial_{Q} \Omega\right)$ назовем относительным вращением поля $\mathscr{F}$ на $\partial_{Q} \Omega$ и обозначим символом $\gamma_{Q}(\mathscr{F}, \Omega)$. Обсуждение свойств вращения $\gamma_{Q}(\mathscr{F}, \Omega)$ можно найти в [5], [8], [9].

Множество $\mathfrak{N} \subset Q$ назьвают особым для поля $\mathscr{F}$ класса $S(Q)$, если любой элемент из $\mathfrak{N}$ есть особая точка $\mathscr{F}$. Особое множество $\mathfrak{N}$ изолировано, если в некоторой его относительной окрестности $O$ нет других особых точек поля $\mathscr{F}$. Для ограниченного изолированного особого множества окрестность $O$ можно взять также ограниченной; в этом случае $\mathfrak{N}$ - компактное множество [9]; относительное вращение $\gamma_{Q}(\mathscr{F}, O)$ назьвается индексом особого множества $\mathfrak{N}$ поля $\mathscr{F}$ и обозначается символом $\operatorname{ind}_{Q}(\mathfrak{N}, \mathscr{F})$.

Основное внимание далее уделяется потенциальным операторам. Напомним некоторые определения. Пусть $f \in \Lambda(Q), x \in Q, v \in X_{0}=\operatorname{Lin}(Q-Q)$. Множество последовательностей $\{y, t\}$ таких, что $y \rightarrow x, t \rightarrow+0,[y, y+t v] \subset Q$, непусто [13]. Положим

$$
f^{0}(x, v)=\varlimsup_{y \rightarrow x, t \rightarrow+0,[y, y+t v] \subset Q} \frac{f(y+t v)-f(y)}{t} .
$$

Число $f^{0}(x, v)$ назьвают обобщенной производной функционала $f$ в точке $x$ по направлению $v$. Функционал $f^{0}(x, \cdot): X_{0} \rightarrow \mathbb{R}$ вьпукл, положительно однороден, удовлетворяет условию Липшица и допускает однозначное продолжение с сохранением указанных свойств на пространство $X=\bar{X}_{0}$. За продолженным функционалом сохраним то же обозначение. Функционал $f^{0}(x, \cdot): X \rightarrow \mathbb{R}$ есть опорная функция множества класса $G\left(X^{*}\right)$, обозначаемого символом $\partial f(x)$ и назьваемого обобщенным градиентом функционала $f$ в точке $x[13],[14]$. Если $f \in \Lambda(Q), x(\cdot):(\alpha, \beta) \rightarrow Q$ - отображение класса $C^{1}$, то для верхней правой производной $D^{*} f(x(t))$ функции $f \circ x:(\alpha, \beta) \rightarrow \mathbb{R}$ в точке $t$ справедлива [15] оценка

$$
D^{*} f(x(t)) \leqslant f^{0}\left(x(t), x^{\prime}(t)\right)
$$

Через $\Lambda_{1}(Q)$ обозначим часть $\Lambda(Q)$, состоящую из функционалов $f$, для которых градиентное отображение $\mathscr{F}(x)=\partial f(x)$ принадлежит классу $S(Q)$. В этом случае функционал $f$ назовем потенциалом оператора $\mathscr{F}$, особые точки $\mathscr{F}$ - критическими точками функционала $f$, неособые точки $\mathscr{F}$ - регулярными точками $f$. Если поверхность уровня $f^{-1}(c)=\{x \in Q, f(x)=c\}$ функционала пересекается с множеством $\mathscr{K}_{f}$ его критических точек, то число с именуют критическим значением $f$; в противном случае $c$-регулярное значение $f$.

Далее используются некоторые понятия алгебраической топологии. Пусть $\mathfrak{R}^{\prime}$ - подпространство метрического пространства $\mathfrak{R}$. Через $H_{l}\left(\mathfrak{R}, \mathfrak{R}^{\prime}\right), l=0,1, \ldots$, обозначается группа относительных целочисленных гомологий с компактными носителями [4], [16], [17] пары пространств $\left(\mathfrak{R}, \mathfrak{R}^{\prime}\right), b_{l}\left(\mathfrak{R}, \mathfrak{R}^{\prime}\right)$ - ранг групшы $H_{l}\left(\mathfrak{R}, \mathfrak{R}^{\prime}\right)$, назьваемьй $l$-м числом Бетти пары $\left(\mathfrak{R}, \mathfrak{R}^{\prime}\right)$. Если $b_{l}\left(\mathfrak{R}, \mathfrak{R}^{\prime}\right)<\infty \forall l$ и $b_{l}\left(\mathfrak{R}, \mathfrak{R}^{\prime}\right)=0$ при $l \geqslant l_{0}$, то число 
$\chi\left(\mathfrak{R}, \mathfrak{R}^{\prime}\right)=b_{0}\left(\mathfrak{R}, \mathfrak{R}^{\prime}\right)+\cdots+(-1)^{l} b_{l}\left(\mathfrak{R}, \mathfrak{R}^{\prime}\right)+\cdots$ называют әйлеровой характеристикой пары $\left(\mathfrak{R}, \mathfrak{R}^{\prime}\right)$. В случае $\mathfrak{R}^{\prime}=\varnothing$ полагают $H_{l}(\mathfrak{R}, \varnothing)=H_{l}(\mathfrak{R}), \chi(\mathfrak{R}, \varnothing)=\chi(\mathfrak{R}), H_{l}(\mathfrak{R})-$ группа абсолютных целочисленных гомологий с компактньми носителями пространства $\mathfrak{R}, \chi(\Re)$ - эйлерова характеристика пространства $\mathfrak{R}$. Если пара $\left(\mathfrak{R}, \mathfrak{R}^{\prime}\right)$ такова, что два из чисел $\chi(\mathfrak{R}), \chi\left(\mathfrak{R}^{\prime}\right), \chi\left(\mathfrak{R}, \mathfrak{R}^{\prime}\right)$ определены, то определено и третье, причем $[16, \S 5.5]$

$$
\chi(\mathfrak{R})-\chi\left(\mathfrak{R}^{\prime}\right)=\chi\left(\mathfrak{R}, \mathfrak{R}^{\prime}\right) .
$$

\section{Существенную роль в последующем играет}

ПРЕДЛОЖЕНИЕ 2 [8]. Пусть $f \in \Lambda_{1}(Q), a$ - регулярное значение функиионала $f$, лебегово множество $M_{a}=\{x \in Q, f(x) \leqslant a\}$ непусто и ограничено. Тогда определена әйлерова характеристика $\chi\left(M_{a}\right)$ пространства $M_{a} u \gamma_{Q}\left(\partial f, M_{a}\right)=\chi\left(M_{a}\right)$.

В этом и следующем пунктах будем предполагать множество $Q$ ограниченньп. Пусть $c$ - единственное критическое значение функционала $f$ класса $\Lambda_{1}(Q)$ на сегменте $[a, b]$, $a<c<b$. В силу предложения 2 имеют смысл эйлеровы характеристики пространств $M_{b}, M_{a}$. Применяя к паре пространств $\left(M_{b}, M_{a}\right)$ равенство $(6)$, получаем $\chi\left(M_{b}, M_{a}\right)=$ $\chi\left(M_{b}\right)-\chi\left(M_{a}\right)$. В частности, при любом целом $l \geqslant 0$ конечны числа Бетти $b_{l}\left(M_{b}, M_{a}\right)$ $(l=0,1, \ldots)$ и последовательность $b_{l}\left(M_{b}, M_{a}\right)$ финитна. Из теоремы 2 работы [8] следует, что числа $b_{l}\left(M_{b}, M_{a}\right)$ не зависят от способа выбора отрезка $[a, b]$, для которого $c \in(a, b)$ и все числа из $[a, b] \backslash\{c\}$ регулярны. Таким образом, числа $b_{l}\left(M_{b}, M_{a}\right)$ характеризуют число $c$. Положим $m_{l}=b_{l}\left(M_{b}, M_{a}\right), l=0,1, \ldots$; определяемые этими равенствами величины $m_{l}(c)$ назовем типовыми числами критического значения $c$.

ТЕОремА 1. Пусть с-изолированное критическое значение функиионала $f$ класса $\Lambda_{1}(Q), \mathfrak{R}_{c}=\mathscr{K}_{f} \cap f^{-1}(c)$ - множество критических точек $f$ на поверхности уровня $f^{-1}(c)$. Тогда

$$
\operatorname{ind}_{Q}\left(\Re_{c}, \partial f\right)=\sum_{l \geqslant 0}(-1)^{l} m_{l}(c) .
$$

ДокАЗАТЕЛЬСтво. Вначале отметим, что $\mathfrak{R}_{c}$ - изолированное критическоемножество, т.е. в некоторой его окрестности нет критических точек функционала $f$. Действительно, в предположении противного существует последовательность $x_{n}$ из $\mathscr{K}_{f}$, для которой $\theta_{X}\left(x_{n}, \mathfrak{R}_{c}\right) \rightarrow 0, f\left(x_{n}\right) \neq c$. Ввиду компактности множества $\mathfrak{R}_{c}$ [9] можно считать, что $x_{n} \rightarrow x$ и $x \in \mathfrak{R}_{c}$. Но тогда $f\left(x_{n}\right) \rightarrow f(x)=c$, а это противоречит изолированности критического значения $c$.

Равенство (6) вытекает из цепи соотношений:

$$
\begin{aligned}
\operatorname{ind}_{Q}\left(\Re_{c}, \partial f\right) & =\gamma_{Q}\left(\partial f, \stackrel{\circ}{M}_{b}\right)-\gamma_{Q}\left(\partial f, \stackrel{\circ}{M}_{a}\right)=\chi\left(M_{b}\right)-\chi\left(M_{a}\right) \\
& =\chi\left(M_{b}, M_{a}\right)=\sum_{l \geqslant 0}(-1)^{l} b_{l}\left(M_{b}, M_{a}\right)=\sum_{l \geqslant 0}(-1)^{l} m_{l}(c),
\end{aligned}
$$

где $a<c<b, c$ - единственное критическое значение функционала $f$ на отрезке $[a, b]$. Последовательно используются теорема об алгебраическом числе особых точек [3], [5], $[12]$, предложение 2 , равенство $\chi\left(M_{b}, M_{a}\right)=\chi\left(M_{b}\right)-\chi\left(M_{a}\right)$, вытекающее из (6), определения эйлеровой характеристики пары $\left(M_{b}, M_{a}\right)$ и типовых чисел $m_{l}(c)$. Теорема доказана. 
Пусть функционал $f$ имеет конечное число критических значений $c_{1}, c_{2}, \ldots, c_{N}$, причем $c_{1}<c_{2}<\cdots<c_{N}$. Очевидно, $c_{1}=\min \{f(x), x \in Q\}$; минимум $f$ на $Q$ достигается [9]. Регулярному значению $b>c_{1}$ функционала $f$ класса $\Lambda_{1}(Q)$ сопоставим многочлены

$$
\mathscr{A}(t)=\sum_{c_{i}<b} \sum_{l \geqslant 0} m_{l}\left(c_{i}\right) t^{l}=\sum_{l \geqslant 0} a_{l} t^{l}, \quad \mathscr{B}(t)=\sum_{l \geqslant 0} b_{l}\left(M_{b}\right) t^{l}
$$

В указанных предположениях имеет место

Теорема 2. Справедливо равенство

$$
\mathscr{A}(t)-\mathscr{B}(t)=(1+t) \mathscr{D}(t)
$$

в котором $\mathscr{D}(t)$ - многочлен с неотрицательными коэффициентами.

ДокАЗАТЕЛЬСтво. Пусть $d_{0}, d_{1}, \ldots, d_{m}$ - последовательность, разделяющая меньшие $b$ критические значения функционала $f$, т.е. $d_{0}<c_{1}<d_{1}<c_{2}<\cdots<d_{m-1}<$ $c_{m}<d_{m}=b$. Рассматривая точную гомологическую последовательность пары $\left(M_{d_{i}}\right.$, $\left.M_{d_{i-1}}\right)$, приходим $[4, \S 16]$ к равенству

$$
\sum_{l \geqslant 0} m_{l}\left(c_{i}\right) t^{l}-\left\{\sum_{l \geqslant 0} b_{l}\left(M_{d_{i}}\right) t^{l}-\sum_{l \geqslant 0} b_{l}\left(M_{d_{i-1}}\right) t^{l}\right\}=(1+t) \mathscr{D}_{i}(t)
$$

где $\mathscr{D}_{i}(t), i=1, \ldots, m,-$ многочлен с неотрицательными коэффициентами. Так как $d_{0}<c_{1}$, то $M_{d_{0}}=\varnothing$. Суммируя полученные равенства по $i$ от 1 до $m$, имеем $\mathscr{A}(t)-\mathscr{B}(t)=$ $(1+t)\left(\mathscr{D}_{1}(t)+\cdots+\mathscr{D}_{m}(t)\right)$. Последнее равенство влечет $(8)$. Теорема доказана.

Непосредственным следствием (8) являются неравенства Морса

$$
\sum_{i=0}^{l}(-1)^{l-i}\left(a_{i}-b_{i}\left(M_{b}\right)\right) \geqslant 0
$$

и их ослабленные варианты: $a_{l} \geqslant b_{l}\left(M_{b}\right), l=0,1, \ldots$ Начиная с некоторого номера $l$, неравенства (9) становятся равенствами; это также вытекает из (8).

2. Топологические характеристики критических точек. В этом пункте вводятся типовые числа критических точек. Ниже используется следуюшее дополнение к предложению 1.

ЛЕмма 1. Пусть $\mathfrak{N}$-множество особых точек оператора $\mathfrak{F}$ класса $S(Q)$. Тогда существуют последовательности $E_{n} \in \Gamma(Q), \delta_{n}>0$ и отобрахсение $w$ класса $\Lambda(Q \backslash \mathfrak{N}, X)$, обладающие свойствами

$$
w(x) \in \operatorname{ri}_{E_{n}}\left(Q \cap E_{n}\right), \quad s(w(x)-x, \mathfrak{F}(x))<-\delta_{n}, \quad x \in Q, \quad \theta_{X}(x, \mathfrak{N}) \geqslant \frac{1}{n} .
$$


ДоКАЗАТЕЛЬСТво. Пусть $f_{0}(x)=\theta_{X}(x, \mathfrak{N}) / \theta_{X}(Q, \mathfrak{N}), x \in Q$. Очевидно, что $f_{0} \in$ $\Lambda(Q), 0 \leqslant f_{0}(x) \leqslant 1(x \in Q), \mathfrak{N}=\left\{x \in Q, f_{0}(x)=0\right\}$. Пусть $\alpha_{n}=1 / 2^{n}, \beta_{n}=3 \alpha_{n}$, $n=1,2, \ldots$ Построим систему функций $\varphi_{n}$ класса $\Lambda(\mathbb{R})$, удовлетворяющих условиям:

$$
\begin{aligned}
& \text { 1) } \quad \varphi_{n}(t) \geqslant 0, \quad \sum_{n=1}^{\infty} \varphi_{n}(t)=1, \quad 0<t \leqslant 1 \\
& \text { 2) } \quad\left\{t: \varphi_{n}(t)>0\right\}=\left(p_{n}, q_{n}\right), \quad \alpha_{n}<p_{n}<q_{n}<\beta_{n}, \quad n=1,2, \ldots .
\end{aligned}
$$

Система интервалов $\left(p_{n}, q_{n}\right), n=1,2, \ldots$, образует открытое покрытие промежутка $(0,1]$ кратности 2 .

Положим $\mathfrak{M}_{n}=\left\{x \in Q, \alpha_{n} \leqslant f_{0}(x) \leqslant \beta_{n}\right\}$. Множество $\mathfrak{M}_{n}$ ограничено, замкнуто и не содержит особых точек поля $\mathfrak{F}$. Согласно предложению 1 существуют $E_{n}^{\prime}$ из $\Gamma(Q)$, поле $w_{n}$ класса $\Lambda(Q, X)$ и постоянная $\delta_{n}^{\prime}>0$ такие, что

$$
w_{n}(x) \in \operatorname{ri}_{E_{n}^{\prime}}\left(Q \cap E_{n}^{\prime}\right) \quad \forall x \in Q, \quad s\left(w_{n}(x)-x, \mathfrak{F}(x)\right)<-\delta_{n}^{\prime} \quad \forall x \in \mathfrak{M}_{n} .
$$

Рассмотрим поле

$$
w(x)=\sum_{n=1}^{\infty} \varphi_{n}\left(f_{0}(x)\right) w_{n}(x), \quad x \in Q
$$

Его сужение на $Q \backslash \mathfrak{N}$ принадлежит классу $\Lambda(Q \backslash \mathfrak{N}, X)$. Соотношения (11) влекут вьполнение свойств (10) при подходящем выборе последовательностей $E_{n}, \delta_{n}(n=1,2, \ldots)$. Лемма доказана.

Применим лемму к потенциальному оператору $\mathfrak{F}$ с потенциалом $f$ класса $\Lambda_{1}(Q)$; в этом случае множество $\mathfrak{N}$ особых точек оператора $\mathfrak{F}=\partial f$ совпадает с множеством $\mathscr{K}_{f}$ критических точек функционала $f$. Фиксируем последовательность $E_{n} \in \Gamma(Q), \delta_{n}>0$ $(n=1,2, \ldots)$, и отображение $w$, обладающие свойствами (10). Отображение $v(x)=$ $w(x)-x\left(x \in Q \backslash \mathscr{K}_{f}\right)$ принадлежит классу $\Lambda\left(Q \backslash \mathscr{K}_{f}, X\right)$ и удовлетворяет вытекающим из (10) соотношениям

$$
v(x) \in \operatorname{ri}_{E_{n}} T_{Q \cap E_{n}}(x), \quad f^{0}(x, v(x))<-\delta_{n}, \quad x \in Q, \quad \theta_{X}\left(x, \mathscr{K}_{f}\right) \geqslant \frac{1}{n} .
$$

При любом $z$ из $Q \backslash \mathscr{K}_{f}$ задача Коши

$$
\frac{d x}{d t}=v(x), \quad x(0)=z
$$

имеет единственное решение $x(t ; z)$, определенное на максимальном промежутке $[0, \omega(z))$. Первое из соотношений $(12)$ гарантирует включение $x(t ; z) \in Q[8],[15]$. Объединяя (12), (13) с (5), приходим к оценке

$$
D^{*} f(x(t ; z)) \leqslant-\delta_{n}, \quad \text { если } \theta_{X}\left(x(t ; z), \mathscr{K}_{f}\right) \geqslant \frac{1}{n} .
$$

Неравенство (14) влечет строгое убьвание функции $f(x(t ; z))$ на $[0, \omega(z))$; скорость этого убывания отделена от нуля на любом отрезке траектории $x=x(t ; z)$, отделенном от множества $\mathscr{K}_{f}$ критических точек функционала $f$. 
Пусть $c \in f\left(\mathscr{K}_{f}\right), \Re_{c}=\mathscr{K}_{f} \cap f^{-1}(c)$ - множество критических точек $f$ на поверхности уровня $f^{-1}(c)$. Предположим, что каждая критическая точка $x_{*}$ из $\mathfrak{R}_{c}$ является изолированной, т.е. в некоторой ее окрестности нет критических точек функционала $f$. Тогда множество $\mathfrak{R}_{c}$ конечно и $c$ - единственное критическое значение $f$ на некотором сегменте $[a, b], a<c<b$.

Фиксируем $z$ из $f^{-1}((c, b])$. Решение $x=x(t ; z)$ задачи Коши (13) определено на некотором промежутке $[0, \omega(z))$. Если $f(x(t ; z)) \geqslant c+\sigma(\sigma>0)$ на каком-то отрезке $[0, T]$, то $D^{*} f(x(t ; z))<-\delta$ с некоторьм $\delta>0$ на том же отрезке. Поэтому множество $\{t>0, f(x(t ; z))>c\}$ есть конечный или бесконечньй интервал $(0, \tau(z))$. Таким образом, для рассматриваемых $z$

$$
f(x(t ; z))>c, \quad 0 \leqslant t<\tau(z), \quad \text { и } \quad \lim _{t \rightarrow \tau(z)-0} f(x(t ; z))=c .
$$

Лемма 2. Пусть $x_{0} \in f^{-1}(c)$. Тогда для каждого $\varepsilon>0$ найдется такое $\delta>0$, что если $z \in Q, b \geqslant f(z)>c u\left\|z-x_{0}\right\|<\delta$, mo $\left\|x(t ; z)-x_{0}\right\|<\varepsilon \quad \forall t \in[0, \tau(z))$.

ДоКАЗАТЕЛЬСТВо. Вначале рассмотрим представляющийся более сложным случай, когда $x_{0} \in \mathfrak{R}_{c}$. Если доказьваемое утверждение неверно, то найдутся такое число $\varepsilon_{0}>0$ и такие последовательности $z_{n} \in Q, t_{n} \in\left[0, \tau\left(z_{n}\right)\right]$, что $b \geqslant f\left(z_{n}\right)>c$, $\left\|z_{n}-x_{0}\right\|<1 / n$ и $\left\|x\left(t_{n} ; z\right)-x_{0}\right\|>\varepsilon_{0}$. Ввиду ограниченности отображения $v$ время $T_{n}$ пребьвания траектории $x=x\left(t ; z_{n}\right)$ вне $\varepsilon_{0} / 2$-окрестности множества $\mathscr{K}_{f}$ ограничено снизу положительной константой: $T_{n} \geqslant T_{0}>0 \forall n$. За это время значение функционала $f$ на траектории $x=x\left(t ; z_{n}\right)$ уменьшится на положительную величину $\varepsilon_{1}$, не зависящую от $n$. Но это противоречит соотношениям $f\left(z_{n}\right) \geqslant f\left(x\left(t ; z_{n}\right)\right)>c\left(0 \leqslant t<\tau\left(z_{n}\right)\right)$, $f\left(z_{n}\right) \rightarrow c$.

Если $x_{0}$ - регулярная точка функционала $f$, то найдется такое $\varepsilon_{0}>0$, что все элементы множества $\left\{x \in Q,\left\|x-x_{0}\right\|<\varepsilon_{0}\right\}$ - также регулярные точки. Теперь доказательство можно провести по той же схеме, что и вьше. Лемма доказана.

ЛЕмма 3. Для любой точки z из $f^{-1}((c, b])$ существует принадлежсащий $f^{-1}(c)$ односторонний предел $\lim _{t \rightarrow \tau(z)-0} x(t ; z)$.

ДокАЗАТЕЛЬСТво. Если $\tau(z)<\infty$, то существование предела вытекает из ограниченности поля $v$ и условия $x^{\prime}=v(x)$. Рассмотрим более сложньй случай $\tau(z)=\infty$. Для любого $\delta>0$ найдется такое число $t$, что $\theta_{X}\left(x(t ; z), \mathscr{K}_{f}\right)<\delta$. Действительно, в противном случае $\theta_{X}\left(x(t ; z), \mathscr{K}_{f}\right) \geqslant \delta \forall t$; отсюда следовало бы, что $f(x(t ; z)) \rightarrow-\infty$ при $t \rightarrow \infty$. Поэтому для каждого натурального числа $n$ найдется такое $t_{n}$, что $\theta_{X}\left(x\left(t_{n} ; z\right)\right.$, $\left.\mathscr{K}_{f}\right)<1 / n$. Некоторая подпоследовательность последовательности $x\left(t_{n} ; z\right)$ сходится к критической точке $x_{0}$ функционала $f$. Отсюда в силу леммы 2 следует, что вся траектория $x=x(t ; z)$ при $t \rightarrow \infty$ сходится к $x_{0}$. Лемма доказана.

Наряду с введенным вьше полем $v=v(x)$ рассмотрим его подправленный вариант $v_{1}(x)=v(x)(f(x)-c)_{+}\left(x \in Q \backslash \mathscr{K}_{f}\right)$ и аналогичную (13) задачу Коши

$$
\frac{d x}{d t}=v_{1}(x), \quad x(0)=z
$$


Если $f(z) \leqslant c$, то решение $x_{1}(t ; z)$ задачи $(15)$ постоянно: $x_{1}(t ; z) \equiv z(t \geqslant 0)$. Для начальных значений $z$ из $f^{-1}((c, b])$ решение $x_{1}(t ; z)$ получается из решения $x(t ; z)$ задачи (13) путем замены времени: справедливо равенство

$$
x_{1}(t ; z)=x(s ; z), \quad s=\int_{0}^{t}\left(f\left(x_{1}(\tau ; z)\right)-c\right) d \tau .
$$

Отметим вытекающую из (15) оценку

$$
\frac{d}{d t}\left(f\left(x_{1}(t ; z)\right)-c\right) \geqslant-k\left(f\left(x_{1}(t ; z)\right)-c\right), \quad 0<k<\infty
$$

приводящую к неравенству $f\left(x_{1}(t ; z)\right)-c \geqslant \exp (-k t)(f(z)-c)$. В частности, $f\left(x_{1}(t ; z)\right)$ $>c$, если $0<t<\infty, c<f(z) \leqslant b$ и $f\left(x_{1}(t ; z)\right) \rightarrow c$ при $t \rightarrow \infty$. Из лемм 2,3 и (16) вытекают леммы 4, 5 .

Лемма 4. Пусть $x_{0} \in f^{-1}(c)$. Тогда для каждого $\varepsilon>0$ найдется такое $\delta>0$, что если $z \in Q, b \geqslant f(z)>c u\left\|z-x_{0}\right\|<\delta$, mo $\left\|x_{1}(t ; z)-x_{0}\right\|<\varepsilon \forall t \in[0, \infty)$.

Лемма 5. Для любой точки z из $f^{-1}((c, b])$ существует принадлежсащий $f^{-1}(c)$ предел $\lim _{t \rightarrow \infty} x_{1}(t ; z)$.

Полезно положить $x_{1}(\infty ; z)=\lim _{t \rightarrow \infty} x_{1}(t ; z)$; согласно лемме $5 x_{1}(\infty ; z) \in f^{-1}(c)$.

Лемма 6. Пусть с-единственное критическое значение функционала $f$ класса $\Lambda_{1}(Q)$ на отрезке $[c, b]$ и каждая критическая точка $x$ из $\mathfrak{R}_{c}$ является изолированной. Тогда множество $M_{c}$ есть деформационный ретракт множества $M_{b}$.

ДоказАТЕЛьство. Введем в рассмотрение отображение $\Psi: M_{b} \times[0,1] \rightarrow M_{b}$, определяемое равенством

$$
\Psi(z, \lambda)=x_{1}\left(\frac{\lambda}{1-\lambda} ; z\right)
$$

в частности, $\Psi(z, 1)=x_{1}(\infty ; z) \in M_{c}$ при любом $z$ из $M_{b}$. Из лемм 4,5 вытекает непрерывность отображения $\Psi$; справедливы соотношения: $\Psi(z, 0)=z\left(z \in M_{b}\right), \Psi(z, \lambda)=z$ $\left(z \in M_{c}, 0 \leqslant \lambda \leqslant 1\right), \Psi(z, 1) \in M_{c}\left(z \in M_{b}\right)$.

Лемма доказана.

Лемма 7. Пусть с-единственное критическое значение функционала $f$ класса $\Lambda_{1}(Q)$ на отрезке $[a, c](a<c)$. Тогда множсество $M_{a}$ есть деформачионный ретракт мнохества $M_{c} \backslash \mathfrak{R}_{c}$.

ДоКАЗАТЕЛЬСТво. Пусть $z \in M_{c} \backslash \mathfrak{R}_{c}$ и $f(z)>a, x(t ; z)$ - решение задачи Коши (13). Функция $f(x(t ; z))$ строго убывает на том отрезке $[0, \beta(z)]$, где $f(x(t ; z)) \geqslant a$. Определим функцию $T(z)$ для $z$ из $\left(M_{c} \backslash \Re_{c}\right) \backslash M_{a}$ исходя из равенства $f(x(T(z) ; z))=a$; для $z$ из $M_{a}$ положим $T(z)=0$. Легко устанавливается непрерьвность функции $T(z)$. Определяемое равенством $\Phi(z, \lambda)=x(\lambda T(z) ; z)$ отображение $\Phi:\left(M_{c} \backslash \mathfrak{R}_{c}\right) \times[0,1] \rightarrow$ $M_{c} \backslash \mathfrak{R}_{c}$ задает искомую деформацию множества $M_{c} \backslash \mathfrak{R}_{c}$ на множество $M_{a}$.

Лемма доказана. 
СЛЕДСТВИЕ. Если выполнены условия лемм 6, 7, то группы $H_{l}\left(M_{b}, M_{a}\right) u$ $H_{l}\left(M_{c}, M_{c} \backslash \mathfrak{R}_{c}\right)$ изоморфны:

$$
H_{l}\left(M_{b}, M_{a}\right) \simeq H_{l}\left(M_{c}, M_{c} \backslash \mathfrak{R}_{c}\right) .
$$

Пусть $x_{0}$ - критическая точка функционала $f$ класса $\Lambda_{1}(Q), c=f\left(x_{0}\right), H_{l}\left(M_{c}\right.$, $\left.M_{c} \backslash x_{0}\right)(l=0,1, \ldots)$ - группа локальных гомологий $[16$, гл. $4, \S 3]$ пространства $M_{c}$ в точке $x_{0}$. Если число $m_{l}\left(x_{0}\right)=\operatorname{rang} H_{l}\left(M_{c}, M_{c} \backslash x_{0}\right)=b_{l}\left(M_{c}, M_{c} \backslash x_{0}\right)<\infty$, то его назьвают $l$-м типовым числом критической точки $x_{0}$. Для любой окрестности $U$ точки $x_{0}$ группа $H_{l}\left(M_{c} \cap U,\left(M_{c} \cap U\right) \backslash x_{0}\right)$ изоморфна группе $H_{l}\left(M_{c}, M_{c} \backslash x_{0}\right)$, поэтому $m_{l}\left(x_{0}\right)=\operatorname{rang} H_{l}\left(M_{c} \cap U,\left(M_{c} \cap U\right) \backslash x_{0}\right)$.

Установим связь между типовыми числами критических значений и критических точек функционала $f$.

Tеорема 3. Пусть $c \in f\left(\mathscr{K}_{f}\right), \mathfrak{R}_{c}=\left\{x_{1}, \ldots, x_{r}\right\}$ - соответствующее критическое множество функиионала $f$ класса $\Lambda_{1}(Q)$, причем каждая из критических точек $x_{i}, i=1, \ldots, r$, изолирована. Тогда типовые числа $m_{l}(c)$ критического значения с и типовые числа $m_{l}\left(x_{i}\right)$ критических точек $x_{i}$ связаны равенством

$$
m_{l}(c)=\sum_{i=1}^{r} m_{l}\left(x_{i}\right), \quad l=0,1, \ldots
$$

ДокАЗАТЕЛЬСТво. Построим произвольные непересекающиеся окрестности $U_{i}$ (относительно пространства $M_{c}$ ) точек $x_{i}$. Используя $(17)$ и вырезая множество $M_{c} \backslash$ $\bigcup_{i=1}^{r} U_{i}$, имеем последовательно

$$
H_{l}\left(M_{b}, M_{a}\right) \simeq H_{l}\left(M_{c}, M_{c} \backslash \Re_{c}\right) \simeq H_{l}\left(\bigcup_{i=1}^{r} U_{i}, \bigcup_{i=1}^{r}\left(U_{i} \backslash x_{i}\right)\right)
$$

Так как окрестности $U_{i}$ не пересекаются, то

$$
H_{l}\left(M_{b}, M_{a}\right) \simeq \bigoplus_{i=1}^{r} H_{l}\left(U_{i}, U_{i} \backslash x_{i}\right)
$$

Теперь (18) вытекает из определения типовых чисел критических значений и критических точек. Теорема доказана.

СлЕДСТВИЕ. Если $\mathfrak{R}_{c}=\left\{x_{0}\right\}$ - единственная изолированная критическая точка функиионала $f$ на поверхности $f^{-1}(c)$, mo $m_{l}(c)=m_{l}\left(x_{0}\right)$ u

$$
\operatorname{ind}_{Q}\left(x_{0}, \partial f\right)=\sum_{l \geqslant 0}(-1)^{l} m_{l}\left(x_{0}\right)
$$

Из $(18)$ следует равенство $m_{l}(c)=m_{l}\left(x_{0}\right)(l \geqslant 0)$. Оно позволяет отождествить (в предположении $\mathfrak{R}_{c}=\left\{x_{0}\right\}$ ) типовые числа критической точки $x_{0}$ с типовыми числами критического значения $c=f\left(x_{0}\right)$. Равенство (19) есть частный случай (7). Оно оказьвается верньп при меньших предположениях относительно числа $c=f\left(x_{0}\right)$. 
ТЕОРемА 4. Пусть $x_{0}$ - изолированная критическая точка функционала $f$ из $\Lambda_{1}(Q)$ и существует последовательность $a_{n}$ регулярных значений $f: a_{n}<f\left(x_{0}\right)$, $a_{n} \rightarrow f\left(x_{0}\right)$. Тогда $m_{l}\left(x_{0}\right)<\infty \quad \forall l, m_{l}\left(x_{0}\right)=0$ при $l \geqslant l_{0}$ и имеет место равенство (19).

ДокАЗАТЕЛЬСТво. Не ограничивая общности, можно считать, что $X, X^{*}$ - локально равномерно вьпуклые пространства. При выполнении этого предположения функционал $f_{0}(x)=\|x\|^{2}$ непрерывно дифференцируем на $X$, его производная $f_{0}^{\prime}$ есть оператор класса $S(X)$ [9]. С помощью подходящей замены переменной можно добиться того, что $x_{0}=0$ есть единственная критическая точка функционала $f$ на множестве $\{x \in Q,\|x\| \leqslant 2\}$. Оператор $\partial f$ невырожден на множестве $\mathscr{P}=\{x \in Q, 1 \leqslant\|x\| \leqslant 2\}$, поэтому найдется такое число $\varepsilon_{0}>0$, что $\theta_{X^{*}}\left(0, \partial f(x)+N_{Q}(x)\right)>2 \varepsilon_{0} \forall x \in \mathscr{P}$.

Фиксируем бесконечно дифференцируемую функцию $\varphi:[0, \infty) \rightarrow \mathbb{R}$, обладающую свойствами: $\varphi(t)=0$, если $0 \leqslant t \leqslant 1 ; \varphi(t)=1$, если $t \geqslant 4 ; 0<\varphi^{\prime}(t)$, если $1<t<4$. Положим $h(x)=\varphi\left(f_{0}(x)\right)(x \in X)$. Отметим следующие свойства функции $h: h \in C^{1}(X)$, $h(x)=0(\|x\| \leqslant 1), h(x)=1(\|x\| \geqslant 2), 0<h(x)<1(1<\|x\|<2), h^{\prime}(x)=$ $\varphi^{\prime}\left(f_{0}(x)\right) f_{0}^{\prime}(x)$.

Покажем, что при любом $\varepsilon>0$ функционал $g_{\varepsilon}=f+\varepsilon h$ принадлежит классу $\Lambda_{1}(Q)$. Справедливо равенство $\partial g_{\varepsilon}(x)=\partial f(x)+\varepsilon h^{\prime}(x)$, из которого вытекает ограниченность оператора $\mathscr{F}=\partial g_{\varepsilon}: Q \rightarrow G\left(X^{*}\right)$. Пусть $x_{n} \in Q, x_{n}^{*} \in \mathscr{F}\left(x_{n}\right)$ - последовательности, обладающие свойствами (1). Положим $z_{n}^{*}=h^{\prime}\left(x_{n}\right), y_{n}^{*}=x_{n}^{*}-\varepsilon z_{n}^{*} \in \partial f\left(x_{n}\right)$. Так как $X$ - рефлексивное пространство, то можно считать, что $z_{n}^{*}$ слабо сходится к некоторому элементу $z^{*} \in X^{*}$. Тогда $y_{n}^{*} \rightarrow y^{*}=x^{*}-\varepsilon z^{*}$ и $\overline{\lim }_{n \rightarrow \infty}\left\langle x_{n}-x, y_{n}^{*}+\varepsilon z_{n}^{*}\right\rangle \leqslant 0$. Прореживая, если это необходимо, последовательность $x_{n}$, можно считать, что $\left\langle x_{n}-x, z_{n}^{*}\right\rangle \rightarrow \gamma_{0}$. Если $\gamma_{0} \geqslant 0$, то имеем: $\varlimsup_{n \rightarrow \infty}\left\langle x_{n}-x, y_{n}^{*}\right\rangle \leqslant 0$. Так как $\partial f \in S(Q)$, то из последнего неравенства получаем: $x_{n} \rightarrow x, y^{*} \in \partial f(x), x^{*}=y^{*}+\varepsilon z^{*} \in \partial f(x)+\varepsilon h^{\prime}(x)=\mathscr{F}(x)$.

Рассмотрим случай $\gamma_{0}<0$. В этом случае последовательность $z_{n}^{*}=h^{\prime}\left(x_{n}\right)$ не стремится к 0; можно считать, что последовательность $\left\|x_{n}\right\|$ отграничена от 1 и $2: 1<R_{1} \leqslant$ $\left\|x_{n}\right\| \leqslant R_{2}<2$. Но $h^{\prime}\left(x_{n}\right)=\varphi^{\prime}\left(f_{0}\left(x_{n}\right)\right) f_{0}^{\prime}\left(x_{n}\right)$; отсюда следует, что $\lim _{n \rightarrow \infty}\left\langle x_{n}-x\right.$, $\left.f_{0}^{\prime}\left(x_{n}\right)\right\rangle \leqslant 0$. Так как $f_{0}^{\prime} \in S(X)$, это снова влечет соотношение $(2)$.

Включение $y_{\varepsilon} \in \Lambda_{1}(Q)$ при любом $\varepsilon>0$ доказано. Положим $\varepsilon=f(0)-a_{n}$. Фиксируем $n$ и $\varepsilon$ так, что $\varepsilon\left\|h^{\prime}(x)\right\|<\varepsilon_{0}(x \in Q,\|x\| \leqslant 2)$. Тогда функционал $g_{\varepsilon}=f+\varepsilon h$ не имеет ненулевых критических точек на множестве $\{x \in Q,\|x\| \leqslant 2\}$. Вне этого множества $h(x)=1, g_{\varepsilon}(x)=f(x)+\varepsilon$. Из равенства $g_{\varepsilon}(x)=f(0)$ следует, что $f(x)=a_{n}, x-$ регулярная точка функционалов $f, g_{\varepsilon}$. Поэтому 0 есть единственная критическая точка функционала $g_{\varepsilon}$ на поверхности уровня $g_{\varepsilon}(x)=f(0)$. Типовые числа точки $x_{0}=0$ для функционалов $f, g_{\varepsilon}$ одинаковы, поскольку эти функционалы совпадают в окрестности данной точки. Имеем последовательно

$$
\operatorname{ind}_{Q}\left(x_{0}, \partial f\right)=\operatorname{ind}_{Q}\left(x_{0}, \partial g_{\varepsilon}\right)=\sum_{l \geqslant 0}(-1)^{l} m_{l}\left(x_{0}\right) .
$$

Теорема доказана.

\section{3. Типовые числа нулевой критической точки однородного функциона-}

ла. Обсудим модификации установленных вьше результатов. Как полагает автор, развитый вьше подход может быть распространен на широкий класс липшицевых функци- 
оналов, определенных на замкнутых подмножествах банахова многообразия. Не затрагивая вопрос с максимальной общностью, рассмотрим случай, когда $Q$ - неограниченное подмножество сепарабельного рефлексивного банахова пространства $X, Q \in G(X)$. Отметим, что если $x \in Q$ и $\|x\|<R$, то нормальные конусы к множествам $Q$ и $Q_{R}=$ $\{z \in Q,\|z\| \leqslant R\}$ совпадают: $N_{Q}(x)=N_{Q_{R}}(x)$. В частности, одинаковы множества удовлетворяющих неравенству $\|x\|<R$ критических точек у функционала $f$ класса $\Lambda_{1}(Q)$ и его сужения на $Q_{R}$. Это позволяет в ряде ситуаций заменить множество $Q$ его ограниченной частью $Q_{R}$ и, следовательно, распространить предшествующие результаты на более общий случай.

Проиллюстрируем сказанное применительно к теоремам 1,2 . Пусть $f \in \Lambda_{1}(Q)$ и при некотором $b<\infty$ множество $M_{b}=\{x \in Q, f(x) \leqslant b\}$ ограничено: $\theta_{X}\left(M_{b}, 0\right)<R$. Тогда включение $x \in M_{b}$ влечет равенство $N_{Q}(x)=N_{Q_{R}}(x)$, поэтому сохраняются соотношения (8), (9).

Определение типовых чисел носит локальньй характер. Оно без всяких изменений переносится на случай неограниченного множества $Q$. Ясно, что если $x_{0}-$ критическая точка функционала $f$ класса $\Lambda_{1}(Q)$ и $\left\|x_{0}\right\|<R$, то $x_{0}$ - критическая точка сужения $f$ на $Q_{R}$ и типовые числа $x_{0}$ как критической точки функционала $f$ и его сужения на $Q_{R}$ одинаковы.

Обсудим способ вычисления типовых чисел однородного функционала. Пусть $Q-$ конус с вершиной в точке 0 . Функционал $f$ класса $\Lambda_{1}(Q)$ назовем $p$-однородным, если $f(\lambda x)=\lambda^{p} f(x)(1<p<\infty, \lambda>0, x \in Q)$; в этом случае $\mathscr{F}=\partial f$ есть $(p-1)$-однородньй оператор класса $S(Q)$. Однородность оператора $\mathscr{F}$ означает, что $\mathscr{F}(\lambda v)=\lambda^{p-1} \mathscr{F}(v)(\lambda>0, v \in Q)$. Справедливы равенства $f^{0}(v, v)=p \cdot f(v)=$ $-f^{0}(v,-v) \quad(v \in Q)$ - естественные аналоги формулы Эйлера $\left\langle v, f^{\prime}(v)\right\rangle=p \cdot f(v)$ для гладкого функционала $f$. Так как $\mathscr{F}(0)=0$, то 0 - критическая точка $p$-однородного функционала $f$; если других критических точек нет, то функционал $f$ назовем невырожденным. Для $p$-однородного функционала $f: Q \rightarrow \mathbb{R}$ лебегово множество $M_{0}=$ $\{x \in Q, f(x) \leqslant 0\}$ коническое: если $x \in M_{0}$, то и $\lambda x \in M_{0}$ при любом $\lambda \geqslant 0$. Оно определяется пересечением с гранищей $\partial B$ шара $B=\{x \in X,\|x\| \leqslant 1\}$.

ЛЕмма 8. Если $f$ - невырожденный р-однородный неотрицательный функционал класса $\Lambda_{1}(Q)$, то $M_{0}\{0\}, M_{0} \cap \partial B=\varnothing$.

ДоКАЗАТЕЛЬСТВо. Если $f\left(x_{0}\right)=0$, то $x_{0}$ - точка минимума функционала $f$ на множестве $Q$. Поэтому $x_{0}$ - критическая точка $f$, а поскольку $f$ - невырожденный функционал, то $x_{0}=0$. Лемма доказана.

СлЕДСТВИЕ. В условиях леммы $8 m_{0}(0)=1, m_{i}(0)=0, i \geqslant 1$.

Рассмотрим теперь случай, когда $\{x \in Q, f(x)<0\} \neq \varnothing$. Группы локальных гомологий пространств $M_{0}$ и $M_{0} \cap B$ в точке 0 совпадают. Обозначим через $f_{1}$ сужение функционала $f$ на $Q_{1}=Q \cap B$. Очевидно, что 0 - изолированная критическая точка функционала $f_{1}$; отличные от 0 критические точки $f_{1}$ могут находиться лишш в множестве $Q \cap \partial B$.

ТЕОРема 5. Пусть $f$-невырожсденный р-однородный функиионал класса $\Lambda_{1}(Q)$ $u\left\{x \in Q, f_{1}(x)<0\right\} \neq \varnothing$. Тогда типовые числа $m_{l}(0)$ нулевой критической точки 
конечны, $m_{l}(0)=0$ при $l \geqslant l_{0}$ и $m_{0}(0)=0$,

$$
m_{1}(0)=b_{0}\left(M_{0} \cap \partial B\right)-1, \quad m_{l}(0)=b_{l-1}\left(M_{0} \cap \partial B\right), \quad l \geqslant 2 .
$$

ДокАЗАТЕЛЬСТво. Установим, что все достаточно малые отрицательные числа являются регулярными значениями функционала $f_{1}: Q_{1} \rightarrow \mathbb{R}$. В предположении противного существует последовательность $x_{n} \in Q \cap \partial B$ критических точек функционала $f_{1}$, для которой $f_{1}\left(x_{n}\right)<0, f_{1}\left(x_{n}\right) \rightarrow 0,0 \in \partial f_{1}\left(x_{n}\right)+N_{Q_{1}}\left(x_{n}\right)$. Нормальный конус $N_{Q_{1}}\left(x_{n}\right)$ есть сумма конусов $N_{Q}\left(x_{n}\right)$ и $N_{B}\left(x_{n}\right)$. Как и при доказательстве теоремы 3 , будем считать, что функционал $f_{0}(x)=\|x\|^{2}$ непрерьвно дифференцируем. Так как $B=\left\{x \in X, f_{0}(x) \leqslant 1\right\}$, то $[14, \S 2.4] N_{B}\left(x_{n}\right)=\bigcup_{\lambda \geqslant 0} \lambda f_{0}^{\prime}\left(x_{n}\right)$. Поэтому справедливо равенство

$$
0=x_{n}^{*}+\lambda_{n} f_{0}^{\prime}\left(x_{n}\right)+y_{n}^{*},
$$

где $x_{n}^{*} \in \partial f\left(x_{n}\right), \lambda_{n} \geqslant 0, y_{n}^{*} \in N_{Q}\left(x_{n}\right)$. Применяя (21) к элементу $x_{n}$, приходим к соотношениям

$$
\begin{gathered}
0=\left\langle x_{n}, x_{n}^{*}\right\rangle+\lambda_{n}\left\langle x_{n}, f_{0}^{\prime}\left(x_{n}\right)\right\rangle+\left\langle x_{n}, y_{n}^{*}\right\rangle=\left\langle x_{n}, x_{n}^{*}\right\rangle+2 \lambda_{n}, \\
2 \lambda_{n} \leqslant-\left\langle x_{n}, x_{n}^{*}\right\rangle \leqslant f^{0}\left(x_{n},-x_{n}\right)=-p f\left(x_{n}\right) .
\end{gathered}
$$

Так как $f\left(x_{n}\right) \rightarrow \infty, \lambda_{n} \geqslant 0$, то $\lambda_{n} \rightarrow 0$. В соединении с (21) это влечет равенство $\lim _{n \rightarrow \infty} \theta_{X^{*}}\left(0, \partial f\left(x_{n}\right)+N_{Q}\left(x_{n}\right)\right)=0$, противоречащее невырожденности функционала $f$.

Таким образом, вьполнены условия теоремы 4 при $x_{0}=0, f\left(x_{0}\right)=0$. Поэтому $m_{l}(0)<\infty \quad \forall l$ и $m_{l}(0)=0$ при $l \geqslant l_{0}$.

Для доказательства равенств (20) заметим, что окрестность $V=M_{0} \cap B$ точки 0 стягиваема, поэтому $[16$, гл. $4, \S 3]$

$$
H_{l}\left(M_{0}, M_{0} \backslash 0\right) \simeq \widetilde{H}_{l-1}(V \backslash 0),
$$

где $\widetilde{H}_{l-1}(V \backslash 0)$ - приведенная гомологическая группа пространства $V \backslash 0[16$, гл. $4, \S 3]$. Но $V \backslash 0$ гомотопически эквивалентно $M_{0} \cap \partial B$, поскольку $M_{0} \cap \partial B$ есть деформационньй ретракт $V \backslash 0$. Следовательно, $H_{l-1}(V \backslash 0) \simeq \widetilde{H}_{l-1}\left(M_{0} \cap \partial B\right)$, что и приводит к равенствам (20).

Теорема доказана.

Комбинация (19), (20) влечет

СледСтвИЕ. В условиях теоремы 5 әйлерова характеристика $\chi\left(M_{0} \cap \partial B\right)$ конечна $u \operatorname{ind}(0, \partial f)=1-\chi\left(M_{0} \cap \partial B\right)$.

Для гладких однородных функций, определенных на конечномерном пространстве $X$, близкое к следствию утверждение анонсировано в [18]. Теорема 5 естественным образом обобщается на функционалы с однородной главной частью.

Автор благодарит рецензента за ценные замечания. 


\section{СПИСОК ЦИТИРОВАННОЙ ЛИТЕРАТУРЫ}

[1] Зейферт Г., Трельфалль В. Вариационное исчисление в целом. М.: ИЛ, 1947.

[2] Скрыпник И. В. Разрешимость и свойства решений нелинейных эллиптических уравнений // Итоги науки и техники. Современные проблемы математики. Т. 9. М.: ВИНИТИ, 1976. C. 131-254.

[3] Скрыпник И. В. Нелинейные эллиптические уравнения высшего порядка. Киев: Наукова думка, 1973.

[4] Дубровин Б. А., Новиков С. П., Фоменко А. Т. Современная геометрия. Методы теории гомологий. М.: Наука, 1984.

[5] Бобылев Н. А., Емельянов С. В., Коровин С. К. Геометрические методы в вариационных задачах. М.: Изд-во "Магистр", 1998.

[6] Похожаев С.И. О разрешимости нелинейных уравнений с нечетными операторами // Функцион. анализ и его прилож. 1967. Т. 1. № 3. С. 66-73.

[7] Browder F. E. Nonlinear elliptic boundary value problems and the generalized topological degree // Bull. Amer. Math. Soc. 1970. V. 76. № 5. P. 999-1005.

[8] Климов В.С. О топологических характеристиках негладких функционалов // Изв. РАН. Сер. матем. 1998. Т. 62. №5. С. 117-134.

[9] Климов В. С., Сенчакова Н. В. Об относительном вращении многозначных потенциальных векторных полей // Матем. сб. 1991. Т. 182. № 10. С. 1393-1407.

[10] Обен ЖК. П., Экланд И. Прикладной нелинейный анализ. М.: Мир, 1988.

[11] Борисович Ю. Г. Об относительном вращении компактных векторных полей в линейньх пространствах // Тр. семинара по функц. анализу. Вып. 12. Воронеж: Изд-во Воронежского ун-та, 1969. С. $3-27$.

[12] Борисович Ю. Г., Гельман Б. Д., Мышкис А. Д. Обуховский В.В. Топологические методы в теории неподвижных точек многозначных отображений // УМН. 1980. Т. 35. № 1. C. 59-126.

[13] Дмитрук А. В., Милютин А. А., Осмоловский Н. П. Теорема Люстерника и теория экстремума // УМН. 1980. Т. 35. №6. С. 11-46.

[14] Кларк Ф. Оптимизация и негладкий анализ. М.: Наука, 1988.

[15] Климов В. С. Минимаксные критические значения негладких функционалов // Сиб. матем. ж. 1992. Т. 33. № 3. С. 91-100.

[16] Дольд А. Лекции по алгебраической топологии. М.: Мир, 1976.

[17] Масси У. Теория гомологий и когомологий. М.: Мир, 1981.

[18] Мухамадиев Э. Ограниченные решения и гомотопические инварианты нелинейных дифференциальных уравнений // Докл. РАН. 1996. Т. 351. № 5. С. 596-598. 